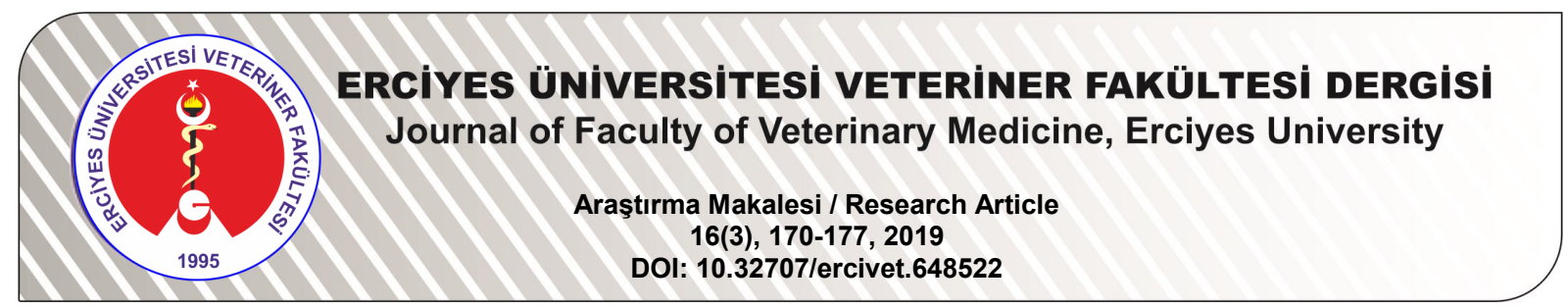

The Effects of Using Oregano and L-Carnitine on Second Phase Laying Performance Parameters of Force Molting Programs in Laying Hens

\author{
Abdil Burhaneddin AKKAYA ${ }^{1}$, İsmail BAYRAM ${ }^{2}$ \\ ${ }^{1}$ CB-İdeal Tarım Hayvancılık Ltd.Şt. Afyonkarahisar-TURKEY \\ ${ }^{2}$ University of Afyon Kocatepe, Faculty of Veterinary Medicine, Department of Animal Nutrition, Afyonkarahisar- TURKEY
}

\begin{abstract}
*Corresponding author: İsmail BAYRAM; E-mail: ibayram1965@gmail.com; ORCID: 0000-0002-9993-7092
How to cite: Akkaya $A B$, Bayram $I$. The effects of using oregano and L-carnitine on second phase laying performance parameters of force molting programs in laying hens. Erciyes Üniv Vet Fak Derg 2019; 16(3): 170-177.
\end{abstract}

\begin{abstract}
Summary: This study was carried out to use Oregano leaf and L-carnitine as an alternative feed additives in alfalfa flour or low Sodium-Calcium (Na-Ca) diet and to determine their effect on second phase laying period after force molting in commercial laying hens. A total of 1170 birds were divided into 13 groups which were further subdivided into 6 replicates having 15 birds in each. A negative control group $(K)$ which was neither forced molted nor provided with any feed additive substances in their diets Moreover, other three treatment groups (M: Marbel powder, $A$ : Alfalfa, F: Low Ca -Na diet), were forced molted and one withhold feeding group was used for the study; the forced molted groups were supplied L-carnitine $(C)(100 \mathrm{ppm})$ and Oregano leaves $(O)(5 \%)$ as a feed additive in designed individual diets (MC, $A C, F C, M O, A O, F O)$ alone and in combination $(50 \mathrm{ppm} ; 2.5 \%)$ in groups (MCO, ACO, FCO). All hens were feeding with commercial feeds during the second laying phase.The trial was 98 days after 22 days of forcing stage. Performance parameters (except egg quality) were measured during 14 days periods. In the results, eggs yield values were found significantly higher $(\mathrm{P}<0.01)$ among the treatment groups over control group. It was concluded that supplementing L-carnitine and Oregano leaves could be used as an alternative feed additives in alfalfa meal during feed withdrawal period and their combination also showed a positive impact on laying performance in laying hens.
\end{abstract}

Key words: Egg yield, L-carnitine, laying hen, force molting, oregano

\title{
Zorlamalı Tüy Dökümü programlarında Kekik ve L-Karnitin Kullanılmasının Yumurta Tavuklarında İkinci Yumurtlama Periyodu Verim Parametreleri Üzerine Etkisi
}

Özet: Bu çalışma, ticari yumurta tavuklarının kekik yaprakları ve L-karnitin katkılı yonca unu veya düşük sodyumkalsiyum (Na-Ca) içeriğine sahip rasyonlarla tüy dökümüne sokulması ve tüy dökümü sonrasında ikinci yumurtlama dönemi verim parametreleri üzerine olan etkilerinin belirlenmesi amacıyla gerçekleştirilmiştir. Deneme, zorlanım uygulanmayan ve yem katkı maddeleri kullanılmayan bir negatif kontrol grubu $(K)$; biri yem çekmeli olmak üzere 3 adet zorlamalı tüy dökümü metodu uygulanan deneme grupları ( $M$ : Mermer tozu, $A$ : Yonca, $F$ : Düşük Na-Ca içerikli yem), zorlanım uygulanan gruplara yem ilavesi olarak L-karnitin $(C)(100 \mathrm{ppm})$ ve kurutulmuş kekik $(O)(\% 5)$ yapraklarının belirlenen oranlarda ayrı ayrı $(M C, A C, F C, M O, A O, F O)$ ve birlikte kullanıldığı $(50$ ppm; \%2,5) gruplar $(M C O, A C O, F C O$, ) olmak üzere, her grupta 6 tekerrür ve tekerrür gruplarında 15 adet hayvan olacak şekilde toplam 1170 adet yumurta tavuğunda ve 13 grupta yürütülmüştür. Denemede kullanılan tüm tavuklar ikinci verim döneminde ticari yumurta tavuğu yemiyle beslenmişlerdir. Araştırma, 22 günlük zorlanım süresi sonrasında 98 gün boyunca sürdürülmüştür. Performans parametreleri (yumurta kalite parametreleri dışında) 14 günlük periyotlar halinde ölçülmüştür. Gruplar arasındaki ortalama yumurta verimleri değerlendirildiğinde, $F C O$ grubu haricinde tüm deneme gruplarının verimleri kontrol grubuna göre önemli ölçüde yüksek bulunmuştur. Araştırmada ölçülen tüm parametreler esas alındığında, sonuç olarak, yonca ununun, geleneksel yem çekme metoduna alternatif olabileceği, yonca unuyla yapılan tüy dökümü yöntemi sonrası verim döneminde rasyona ilave olarak L-karnitin ve kurutulmuş kekik yapraklarının bir arada katılmasının performans üzerine olumlu etkiler gösterebileceği kanısına varıımıştır.

Anahtar Kelimeler: Kekik, L-karnitin, yumurta tavuğu, yumurta verimi, zorlamalı tüy dökümü

\section{Introduction}

Forced molting is a method used to prolong the productive life of broiler and laying hens or to interrupt egg yield temporarily when egg prices are very low. Various methods are used for molting such as re-

Geliş Tarihi/Submission Date : 26.12.2017 Kabul Tarihi/Accepted Date : 11.02 .2019 stricting feed, restricting water and light, providing feed with inadequate calcium or sodium, adding elements such as aluminum, zinc and iodine, feeding only grain or cassava and administering various drugs and hormones $(4,12,25)$. The traditional method of removing feed and water together or alone is the most commonly used method of short-term forced molting. Following the forced molting, a number of 
Table 1. Composition of the experimental diets, $\mathrm{kg} / \mathrm{ton}$

\begin{tabular}{lcc}
\hline Feedstuffs & $\begin{array}{c}\text { Negative control group (K)\& second } \\
\text { laying period ration of all groups }\end{array}$ & Low Na-Ca Diets (F groups) \\
\hline Corn grain & 371.81 & 381.33 \\
Wheat grain & 174.00 & 220.00 \\
Sunflower meal CP 36\% & 140.00 & 0.00 \\
Sunflower meal CP 28\% & 0.00 & 150.00 \\
Barley grain & 0.00 & 30.00 \\
Rasmol & 30.00 & 90.00 \\
Soybean meal CP 48\% & 34.00 & 94.00 \\
Full fat soy & 44.00 & 0.00 \\
Canola meal & 30.00 & 0.00 \\
Corn bran & 30.00 & 0.00 \\
DDGS (Corn) & 30.00 & 0.00 \\
Sunflower oil & 10.00 & 0.00 \\
Dicalcium phosphate & 6.74 & 7.50 \\
Marbel powder & 89.00 & 20.00 \\
Salt & 3.50 & 0.00 \\
L-Lysine HCl & 1.10 & 1.15 \\
DL-Methionine & 0.70 & 0.92 \\
Safizyme XP 20 & 1.00 & 1.00 \\
Karzyme P 500 & 0.65 & 0.60 \\
Kavimix 23 15/5** & 2.50 & 2.50 \\
Kavimix M 1 & 1.00 & 1.00 \\
Analysed values & & \\
Crude protein\% & 16.47 & 16.88 \\
Crude cellulose \% & 5.01 & 6.06 \\
Crude ash \% & 12.54 & 6.93 \\
Crude fat \% & 4.27 & 3.14 \\
Dry Matter \% & 88.58 & 88.53 \\
\hline
\end{tabular}

* Endo-1,4-beta-xylanase, Kartal Kimya, Turkey: $1.400 .000 \mathrm{U}$

** Phytase, Kartal Kimya, Turkey: 500.000 FTU/g

${ }^{* * *}$ Vitamin Premix, Kartal Kimya, Turkey: Each 2,5 kg Vitamin A: 12.000 .000 IU, Vitamin $D_{3}: 2.400 .000$ IU, Vitamin E: 30.000 $\mathrm{mg}$, Vitamin $\mathrm{K}_{3}: 2.500 \mathrm{mg}$, Vitamin $B_{1}: 3.000 \mathrm{mg}$, Vitamin $B_{2}: 7.000 \mathrm{mg}$, Vitamin $B_{6}: 4.000 \mathrm{mg}$, Vitamin $B_{12}: 15 \mathrm{mg}$, Niacin: $40.000 \mathrm{mg}$, Ca-D-Pantothenate: $8.000 \mathrm{mg}$, Folic Acid: $1.000 \mathrm{mg}$, D-Biotine: $45 \mathrm{mg}$, Vitamin C :50.000 mg, Choline Cl. : $125.000 \mathrm{mg}$, Canthaxantin: $1.500 \mathrm{mg}$, Apo-Carotenoic acid ester: $500 \mathrm{mg}$ include.

****Mineral Premix, Kartal Kimya, Turkey: Each 1 kg Manganese: 80.000 mg, Iron: 40.000 mg, Zinc: 60.000 mg, Copper: 5000 $\mathrm{mg}$, l̇odine: $400 \mathrm{mg}$, Cobalt: $100 \mathrm{mg}$ Selenium: $150 \mathrm{mg}$ include.

researchers have reported that eggs obtained in the second yield stage are heavier than those obtained in the first yield, an increase in shell breaking strength, an improvement in egg quality, especially in the Haugh unit $(2,11,16)$. However, in forced-molted chickens, there are also studies of similar egg weight, yellow index and egg yolk color as well as non- molted chickens (27). It has been reported that alternative forced-molting methods can provide better living conditions for the birds and these chickens can go through the production period more comfortably during the rest period (19). Minoura et al. (21) reported that force molting made with wheat or rice bran improves egg production. In a study using forced molting methods, it has been shown that giving vitamin $\mathrm{E}$ to laying hens positively affects the immunity system (13). The number of studies dealing with the applicability of the oregano and L-carnitine and their products for forced molting are limited. In view of this in- formation, due to the fact that conventional withdrawal forced molting method is not suitable for animal welfare. Therefore, this study has been carried out to comparison of alternative non feed withdrawal molting methods and withdrawal (fasting) methods in laying hens.

\section{Material and Methods}

A total of 1170 white laying hens (Lohmann LSL), 72 weeks old were used in this study. The study was carried out in the poultry unit of Kocatepe University Livestock Research Center (KUHAM). The experimental protocols were approved by the Animal Care and Ethical Committee at Afyon Kocatepe University (147-2008). The experimental rations (standard commercial laying hen feeds) was used during the adaptation and egg laying periods and also fed to the negative control group during the trial (molting phase) period. The feed was formulated according to NRC 
Table 2. Experimental groups in force molting period

\begin{tabular}{|c|c|c|c|c|c|c|c|}
\hline & & \multicolumn{2}{|c|}{ Molting Method } & \multicolumn{2}{|c|}{ Feed Additives } & \multicolumn{2}{|c|}{ Method\&Water } \\
\hline Groups & $\begin{array}{l}\text { Group } \\
\text { Name }\end{array}$ & $0-11$ day & 12-22 day & L-Carnitine $\mathrm{HCl}$ & Oregano & Method & Water \\
\hline 01 & $\mathrm{~K}$ & $\mathrm{Neg}$ & tive Control & No & No & No & Ad-libitum \\
\hline 02 & M & & & No & No & & \\
\hline 03 & $\mathrm{MC}$ & & & 100 ppm & No & & \\
\hline 04 & MO & $\begin{array}{l}\text { Marbel } \\
\text { powder }\end{array}$ & $\begin{array}{l}\text { Limited Barley } \\
\text { grain }\end{array}$ & No & $5 \%$ & withdrawal & Ad-libitum \\
\hline 05 & MCO & & & $50 \mathrm{ppm}$ & $2.5 \%$ & & \\
\hline 06 & A & & & No & No & & \\
\hline 07 & $A C$ & & & 100 ppm & No & & \\
\hline 08 & $\mathrm{AO}$ & $\begin{array}{l}\text { Alfalfa } \\
\text { powder }\end{array}$ & Alfalfa powder & No & $5 \%$ & No withdrawal & Ad-libitum \\
\hline 09 & $\mathrm{ACO}$ & & & 50 ppm & $2.5 \%$ & & \\
\hline 10 & $\mathrm{~F}$ & & & No & No & & \\
\hline 11 & FC & & & 100 ppm & No & & \\
\hline 12 & FO & $\begin{array}{c}\text { Low } \mathrm{Na}, \mathrm{Ca} \\
\text { Feed }\end{array}$ & $\begin{array}{c}\text { Low } \mathrm{Na}, \mathrm{Ca} \\
\text { Feed }\end{array}$ & No & $5 \%$ & No withdrawal & Ad-libitum \\
\hline 13 & $\mathrm{FCO}$ & & & 50 ppm & $2.5 \%$ & & \\
\hline
\end{tabular}

K:Negative control group, M: Marbel powder, MC: Marbel powder +Carnitine, MO: Marbel powder +Oregano, MCO: Marbel powder +Carnitine+Oregano A:Alfalfa, AC: Alfalfa+Carnitine, AO: Alfalfa+Oregano, ACO: Alfalfa+Oregano+ Carnitine F:Low $\mathrm{Na}-\mathrm{Ca}$, FC: Low Na-Ca+ Carnitine, FO: Low Na-Ca+Oregano, FCO: Low Na-Ca+ Oregano+ Carnitine

(23) (Table 1) and all experimental groups are shown in Table 2. The alfalfa and dried Oregano leaf (Oreganum onites) were used as powder form in the study. The alfalfa was obtained from AfyonkarahisarTurkey as mixture of 1.2. and 3. cuttings. Oregano leaf was purchased from Herba Gida Ltd, İzmirTurkey. L-carnitine was used as a premix with 50.000 mg L-carnitine HCL per one kilogram. There were 13 groups in the study. One was negative control group $(K)$ and the others were trial groups $(M, M C M O$, MCO, A, AC, AO, ACO, F, FC, FO, FCO). There were three main groups; 1-Marbel powder $(M)$, 2Alfalfa $(A), 3$-Low $\mathrm{Na}$ and $\mathrm{Ca}(F)$ respectively in the study. While feed withdrawal method applied to the marbel groups $(M, M C, M O, M C O)$, it was not applied to the other groups ( $A, A C, A O, A C O, F, F C, F O$, $F C O)$. The groups were named according to whether they had received feed additives or not. The groups subjected to force molting were given L-carnitine $(C)$ and Oregano leaf powder $(O)$ as a feed additive separately $(M C, A C, F C, M O, A O, F O)$ and together (MCO, ACO, FCO) to 6 replicas in each group having 15 animals in the replica groups (Table 2). The hens were distributed randomly into an apartment type cage system $(50 \mathrm{~cm}$ length $\times 60 \mathrm{~cm}$ width $\times 56 \mathrm{~cm}$ high) with 5 animals in each cage. The study was performed in three different stages. The first 2 weeks was adaptation period, then 22 days was forced molting and the second laying period contained 14 weeks egg-laying period. During the forced molting period the molting trial groups were subjected to light restriction (there was no used artificial light) while the negative control group was kept under the same environmental conditions with no light restriction and ensured 16 hours of light in their cages within the unit. All hens were provided ad libitum feed and water throughout the last period. The molting groups were given $100 \mathrm{ppm}$ of L-carnitine $\mathrm{HCl}$ and $5 \%$ dried Oregano separately and $50 \mathrm{ppm}$ of L-carnitine $\mathrm{HCl}$ and $2.5 \%$ dried Oregano together. During the study, deaths were recorded on a daily basis while molting period viability and yield period viability were calculated as percentage. Egg yields were recorded daily throughout the yield period for fourteen weeks while feed consumption and egg weights were recorded once every 14 days. At the beginning and end of the yield period, 3 eggs were collected from each subgroup (total 18 eggs) which were examined for egg shape index, egg weight, Haugh units, eggshell thickness and yolk color as an egg quality parameters $(11,27)$. The feed used in the trial was analyzed according to AOAC procedures (3) (Table 1). Statistical analyses of the collected data were performed using one-way analysis of variance in the SAS statistical program (26) package program. Tukey test was applied on the data to assess importance.

\section{Results}

It was found that egg yield mean values showed a difference between the groups in whole yield period including the second yield period and the yields of all the experimental groups except FCO group were found significantly $(P<0.05)$ higher than the control group (Table 3 ). When the study is assessed as a whole, it was observed that the egg weight varied significantly $(P<0.05)$ between groups. Regarding feed consumption, it is observed that group ACO 
Table 3. Egg production and egg weights of groups in the second laying cycle (0-98 days)

\begin{tabular}{|c|c|c|c|c|c|c|c|}
\hline $\begin{array}{l}\text { Gro- } \\
\text { ups }\end{array}$ & $\begin{array}{l}\text { Egg produc- } \\
\text { tion \% } \\
\text { Molting sta- } \\
\text { ge } \\
11-22 \text {.day } \\
\text { Mean } \pm \text { SEM }\end{array}$ & $\begin{array}{l}\text { Egg pro- } \\
\text { duction \% } \\
\text { After } \\
\text { molting } \\
\text { 0-98.day } \\
\text { Mean } \pm \text { SEM }\end{array}$ & $\begin{array}{l}\text { Feed con- } \\
\text { sumption } \\
\text { (g/day) } \\
\text { Mean } \pm S E \\
\text { M }\end{array}$ & $\begin{array}{l}\text { Feed con- } \\
\text { version ratio } \\
(\mathrm{kg} \mathrm{feed/kg} \\
\text { egg mass) } \\
\text { Mean } \pm \text { SEM }\end{array}$ & $\begin{array}{l}\text { Mean } \pm S E \\
M\end{array}$ & $\begin{array}{l}\text { Mortality } \\
\text { rate } \\
\text { Molting } \\
\text { stage } \\
(0-22 \\
\text { days }) \\
\text { Mean } \pm S E \\
\text { M }\end{array}$ & $\begin{array}{l}\text { Mortality rate } \\
\text { Second produc- } \\
\text { tion cycle (0-98 } \\
\text { days) } \\
\text { Mean士SEM }\end{array}$ \\
\hline $\mathrm{K}$ & $65.98^{\mathrm{a}} \pm 1.47$ & $\begin{array}{l}64.38^{\mathrm{e}} \pm 0.4 \\
5\end{array}$ & $\begin{array}{l}132.53^{\mathrm{abc}} \pm \\
2.23\end{array}$ & $1.98^{\mathrm{cd}} \pm 0.03$ & $\begin{array}{l}66.97^{\mathrm{a}} \pm 0 . \\
27\end{array}$ & $\begin{array}{l}2.22^{\mathrm{bc}} \pm 1.4 \\
1\end{array}$ & $1.05 \pm 0.40$ \\
\hline M & $0.00^{d} \pm 0.00$ & $\begin{array}{l}83.18^{\mathrm{a}} \pm 0.4 \\
8\end{array}$ & $\begin{array}{l}135.55^{\mathrm{ab}} \pm \\
1.87\end{array}$ & $2.09^{a b c} \pm 0.03$ & $\begin{array}{l}64.90^{\text {bcde }} \\
\pm 0.29\end{array}$ & $\begin{array}{l}14.39^{\mathrm{ab}} \pm 3 \\
62\end{array}$ & $1.45 \pm 0.47$ \\
\hline MC & $0.00^{d} \pm 0.00$ & $\begin{array}{l}84.24^{\mathrm{a}} \pm 0.5 \\
0\end{array}$ & $\begin{array}{l}132.14^{\mathrm{abc}} \pm \\
2.26\end{array}$ & $2.03^{\text {bcd }} \pm 0.03$ & $\begin{array}{l}65.07^{\mathrm{bcd}} \pm \\
0.34\end{array}$ & $\begin{array}{l}12.22^{\mathrm{abc}} \pm 2 \\
.05\end{array}$ & $0.98 \pm 0.42$ \\
\hline MO & $0.00^{d} \pm 0.00$ & $\begin{array}{l}83.77^{\mathrm{a}} \pm 0.4 \\
8\end{array}$ & $\begin{array}{l}134.93^{\mathrm{ab}} \pm \\
1.64\end{array}$ & $2.07^{\mathrm{abc}} \pm 0.03$ & $\begin{array}{l}65.09^{\mathrm{bcd}} \pm \\
0.38\end{array}$ & $\begin{array}{l}8.89^{a b c} \pm 2 \\
81\end{array}$ & $0.38 \pm 0.27$ \\
\hline $\mathrm{MCO}$ & $0.00^{d} \pm 0.00$ & $\begin{array}{l}80.44^{b c} \pm 0.4 \\
3\end{array}$ & $\begin{array}{l}134.99^{\mathrm{ab}} \pm \\
2.44\end{array}$ & $2.09^{\mathrm{abc}} \pm 0.04$ & $\begin{array}{l}64.47^{\text {cde }_{ \pm}} \\
0.41\end{array}$ & $\begin{array}{l}10.00^{\mathrm{abc}} \pm 5 \\
.64\end{array}$ & $0.46 \pm 0.33$ \\
\hline A & $0.00^{\mathrm{d}} \pm 0.00$ & $\begin{array}{l}80.37^{c} \pm 0.5 \\
7\end{array}$ & $\begin{array}{l}133.65^{\mathrm{abc}} \pm \\
2.06\end{array}$ & $2.09^{a b c} \pm 0.03$ & $\begin{array}{l}63.99^{\text {cde }} \\
0.40\end{array}$ & $\begin{array}{l}10.00^{\mathrm{abc}} \pm 1 \\
.49\end{array}$ & $2.71 \pm 0.97$ \\
\hline$A C$ & $0.00^{d} \pm 0.00$ & $\begin{array}{l}82.86^{\mathrm{ab}} \pm 0.6 \\
6\end{array}$ & $\begin{array}{l}134.47^{\mathrm{abc}} \pm \\
1.53\end{array}$ & $2.07^{\mathrm{abc}} \pm 0.02$ & $\begin{array}{l}65.01^{\mathrm{bcd}} \pm \\
0.27\end{array}$ & $\begin{array}{l}12.22^{\mathrm{abc}} \pm 1 \\
.11\end{array}$ & $1.23 \pm 0.68$ \\
\hline $\mathrm{AO}$ & $0.00^{\mathrm{d}} \pm 0.00$ & $\begin{array}{l}82.77^{\mathrm{abc}} \pm 0 . \\
56\end{array}$ & $\begin{array}{l}135.60^{\mathrm{ab}} \pm \\
2.41\end{array}$ & $2.13^{\mathrm{ab}} \pm 0.04$ & $\begin{array}{l}63.61^{\mathrm{de}} \pm 0 \\
.42\end{array}$ & $\begin{array}{l}12.22^{\mathrm{abc}} \pm 2 \\
.05\end{array}$ & $1.41 \pm 0.70$ \\
\hline $\mathrm{ACO}$ & $0.00^{\mathrm{d}} \pm 0.00$ & $\begin{array}{l}85.19^{a} \pm 0.5 \\
8\end{array}$ & $\begin{array}{l}139.98^{\mathrm{a}} \pm 2 . \\
13\end{array}$ & $2.21^{\mathrm{a}} \pm 0.03$ & $\begin{array}{l}63.43^{\mathrm{e}} \pm 0 . \\
36\end{array}$ & $\begin{array}{l}15.56^{\mathrm{a}} \pm 4.4 \\
4\end{array}$ & $1.78 \pm 0.73$ \\
\hline$F$ & $34.06^{b c} \pm 1.52$ & $\begin{array}{l}71.45^{\mathrm{d}} \pm 0.5 \\
1\end{array}$ & $\begin{array}{l}124.99^{c} \pm 1 \\
76\end{array}$ & $1.91^{\mathrm{d}} \pm 0.03$ & $\begin{array}{l}65.37^{\mathrm{bc}} \pm 0 \\
.26\end{array}$ & $\begin{array}{l}3.34^{\mathrm{abc}} \pm 1 \\
49\end{array}$ & $1.41 \pm 0.52$ \\
\hline $\mathrm{FC}$ & $32.81^{\mathrm{c}} \pm 1.56$ & $\begin{array}{l}70.29^{d} \pm 0.5 \\
1\end{array}$ & $\begin{array}{l}130.02^{\mathrm{bc}} \pm 2 \\
.06\end{array}$ & $1.96^{\mathrm{cd}} \pm 0.03$ & $\begin{array}{l}66.38^{\mathrm{ab}} \pm 0 \\
.27\end{array}$ & $1.11^{\mathrm{c}} \pm 1.11$ & $1.39 \pm 0.51$ \\
\hline $\mathrm{FO}$ & $37.68^{b} \pm 1.97$ & $\begin{array}{l}71.05^{\mathrm{d}} \pm 0.4 \\
6\end{array}$ & $\begin{array}{l}130.85^{\mathrm{abc}} \pm \\
1.55\end{array}$ & $1.95^{\mathrm{cd}} \pm 0.02$ & $\begin{array}{l}67.03^{\mathrm{a}} \pm 0 . \\
27\end{array}$ & $0.00^{c} \pm 0.00$ & $1.37 \pm 0.50$ \\
\hline $\mathrm{FCO}$ & $34.08^{\mathrm{bc}} \pm 1.71$ & $\begin{array}{l}69.90^{\mathrm{de}} \pm 0.5 \\
9\end{array}$ & $\begin{array}{l}128.61^{\mathrm{bc}} \pm 2 \\
.04\end{array}$ & $1.95^{\mathrm{cd}} \pm 0.03$ & $\begin{array}{l}66.02^{\mathrm{ab}} \pm 0 \\
.27\end{array}$ & $\begin{array}{l}2.22^{\mathrm{bc}} \pm 1.4 \\
1\end{array}$ & $1.23 \pm 0.43$ \\
\hline$P$ & 0.001 & 0.001 & 0.001 & 0.001 & 0.007 & 0.001 & 0.451 \\
\hline
\end{tabular}

a,b,c,d,e: Means with different superscripts in each row are significantly different

consume feed significantly $(P<0.05)$ higher however, group $F$ consume significantly $(P<0.05)$ lower feed consumption as compared with other treatment and control group (Table 3). Feed conversion ratio (FCR) vary between 1.91 and 2.21 and significant $(P<0.05)$ differences were found between the mean values of the groups (Table 3 ). Mortality rate remained nonsignificant $(P>0.05)$ (Table 3$)$ between the groups in the study period between 0-98 days. For egg quality parameters, no significant $(P>0.05)$ difference was observed between the groups in the first and last analysis except for egg yolk color $(P>0.05)$ (Table 4$)$.

\section{Discussion}

The findings of our study coincide with the statements made that forced molting has a positive impact on egg yield $(6,7,14)$. An assessment of the egg yield during the yield period reveals that group ACO $(85.19 \%)$ showed significantly high egg yields while the lowest yields were manifested by group FCO
(69.90\%). Aygun and Yetisir (5) found the same results in their work, that their results were similar between the groups during 40 weeks period. It is concluded that the egg yield obtained in present study was much higher than the values reported by Kucukyilmaz et al. (20) for poultry subjected to different molting periods and the egg yield values reported by Keshavarz and Quimby (15) for a group molted with the application of the feed withdrawal method. Koelkebek and Anderson (18) reported a similar egg yield performance during a 10-day feed withdrawal method and withdrawal feed contained maize-soya shells and maize-wheat chips. An examination of the egg yields of groups provided alfalfa flour during 0-98 days of the study reveals that alfalfa flour supplemented groups have similar yields as $M, M C$, and $M O$ whereas the yield of the alfalfa flour group without supplements was lower but similar to group $M C O$. Therefore, it can be asserted that alfalfa flour supplemented with L-carnitine and/or Oregano can be used 
Table 4. Egg quality parameters (Initial and end of experiment)

\begin{tabular}{|c|c|c|c|c|c|c|c|c|}
\hline \multirow{2}{*}{$\begin{array}{l}\text { Gro- } \\
\text { ups }\end{array}$} & \multicolumn{4}{|c|}{ Initial of experiment } & \multicolumn{4}{|c|}{ End of experiment } \\
\hline & $\begin{array}{l}\text { Egg shape } \\
\text { Index }(\%) \\
\text { Mean } \pm \text { SEM }\end{array}$ & $\begin{array}{l}\text { Egg Shell } \\
\text { Thickness } \\
\text { mm } \\
\text { Mean } \pm S E \\
M\end{array}$ & $\begin{array}{l}\text { Mean } \pm \text { SE } \\
M\end{array}$ & $\begin{array}{l}\text { Egg Yolk } \\
\text { Color }\end{array}$ & $\begin{array}{l}\text { Egg sha- } \\
\text { pe index } \\
(\%) \\
\text { Mean } \pm S \\
\text { EM }\end{array}$ & $\begin{array}{l}\text { Egg Shell } \\
\text { Thick- } \\
\text { ness, } \\
\mathrm{mm} \\
\text { Mean } \pm S E \\
\text { M }\end{array}$ & $\begin{array}{l}\text { Mean } \pm S \\
\text { EM }\end{array}$ & $\begin{array}{l}\text { Egg } \\
\text { Yolk } \\
\text { Color }\end{array}$ \\
\hline $\bar{K}$ & $72.78 \pm 0.48$ & $0.35 \pm 0.006$ & $\begin{array}{l}67.01 \pm 0.2 \\
8\end{array}$ & $\begin{array}{l}10.61^{\mathrm{ab}} \pm 0.2 \\
4\end{array}$ & $\begin{array}{l}72.06^{\mathrm{b}} \pm 0 \\
.73\end{array}$ & $\begin{array}{l}0.34 \pm 0.00 \\
6\end{array}$ & $\begin{array}{l}67.44 \pm 0 . \\
31\end{array}$ & $\begin{array}{l}11.78^{\mathrm{a}} \pm \\
0.13\end{array}$ \\
\hline M & $74.94 \pm 0.37$ & $0.35 \pm 0.004$ & $\begin{array}{l}67.21 \pm 0.2 \\
0\end{array}$ & $11.67^{a} \pm 0.21$ & $\begin{array}{l}74.89^{\mathrm{a}} \pm 0 \\
.61\end{array}$ & $\begin{array}{l}0.34 \pm 0.00 \\
6\end{array}$ & $\begin{array}{l}68.50 \pm 0 . \\
29\end{array}$ & $\begin{array}{l}11.17^{\mathrm{ab}} \pm \\
0.19\end{array}$ \\
\hline MC & $74.67 \pm 0.68$ & $0.35 \pm 0.004$ & $\begin{array}{l}67.40 \pm 0.1 \\
7\end{array}$ & $\begin{array}{l}11.50^{\mathrm{ab}} \pm 0.2 \\
2\end{array}$ & $\begin{array}{l}73.67^{\mathrm{ab}} \pm \\
0.59\end{array}$ & $\begin{array}{l}0.34 \pm 0.00 \\
6\end{array}$ & $\begin{array}{l}68.55 \pm 0 . \\
26\end{array}$ & $\begin{array}{l}11.67^{\mathrm{ab}} \pm \\
0.20\end{array}$ \\
\hline $\mathrm{MO}$ & $74.39 \pm 0.44$ & $0.34 \pm 0.005$ & $\begin{array}{l}67.80 \pm 0.1 \\
6\end{array}$ & $\begin{array}{l}10.78^{\mathrm{ab}} \pm 0.1 \\
9\end{array}$ & $\begin{array}{l}73.50^{\mathrm{ab}} \pm \\
0.62\end{array}$ & $\begin{array}{l}0.34 \pm 0.00 \\
5\end{array}$ & $\begin{array}{l}68.62 \pm 0 . \\
25\end{array}$ & $\begin{array}{l}11.44^{\mathrm{ab}} \pm \\
0.17\end{array}$ \\
\hline $\begin{array}{l}M C \\
O\end{array}$ & $74.33 \pm 0.61$ & $0.35 \pm 0.006$ & $\begin{array}{l}67.60 \pm 0.2 \\
5\end{array}$ & $\begin{array}{l}10.78^{\mathrm{ab}} \pm 0.2 \\
4\end{array}$ & $\begin{array}{l}74.06^{\mathrm{ab}} \pm \\
0.57\end{array}$ & $\begin{array}{l}0.34 \pm 0.00 \\
5\end{array}$ & $\begin{array}{l}68.24 \pm 0 . \\
22\end{array}$ & $\begin{array}{l}11.22^{\mathrm{ab}} \pm \\
0.19\end{array}$ \\
\hline$A$ & $74.61 \pm 0.24$ & $0.35 \pm 0.007$ & $\begin{array}{l}66.88 \pm 0.2 \\
3\end{array}$ & $\begin{array}{l}11.50^{\mathrm{ab}} \pm 0.3 \\
3\end{array}$ & $\begin{array}{l}74.33^{\mathrm{ab}} \pm \\
0.54\end{array}$ & $\begin{array}{l}0.35 \pm 0.00 \\
5\end{array}$ & $\begin{array}{l}68.78 \pm 0 . \\
46\end{array}$ & $\begin{array}{l}11.78^{\mathrm{a}} \pm \\
0.19\end{array}$ \\
\hline$A C$ & $73.22 \pm 0.50$ & $0.35 \pm 0.006$ & $\begin{array}{l}67.03 \pm 0.2 \\
4\end{array}$ & $\begin{array}{l}11.56^{\mathrm{ab}} \pm 0.1 \\
8\end{array}$ & $\begin{array}{l}74.39^{\mathrm{ab}} \pm \\
0.54\end{array}$ & $\begin{array}{l}0.34 \pm 0.00 \\
5\end{array}$ & $\begin{array}{l}68.71 \pm 0 . \\
25\end{array}$ & $\begin{array}{l}11.00^{\mathrm{ab}} \pm \\
0.23\end{array}$ \\
\hline $\mathrm{AO}$ & $73.83 \pm 0.56$ & $0.33 \pm 0.003$ & $\begin{array}{l}66.70 \pm 0.3 \\
1\end{array}$ & $11.06^{a} \pm 0.15$ & $\begin{array}{l}74.22^{\mathrm{ab}} \pm \\
0.48\end{array}$ & $\begin{array}{l}0.34 \pm 0.00 \\
7\end{array}$ & $\begin{array}{l}67.76 \pm 0 . \\
30\end{array}$ & $\begin{array}{l}11.33^{\mathrm{ab}} \pm \\
0.18\end{array}$ \\
\hline $\mathrm{ACO}$ & $74.06 \pm 0.69$ & $0.35 \pm 0.004$ & $\begin{array}{l}67.32 \pm 0.2 \\
4\end{array}$ & $10.67^{b} \pm 0.30$ & $\begin{array}{l}74.17^{\mathrm{ab}} \pm \\
0.60\end{array}$ & $\begin{array}{l}0.34 \pm 0.00 \\
6\end{array}$ & $\begin{array}{l}68.59 \pm 0 . \\
26\end{array}$ & $\begin{array}{l}10.89^{b} \pm \\
0.20\end{array}$ \\
\hline$F$ & $73.17 \pm 0.96$ & $0.34 \pm 0.003$ & $\begin{array}{l}67.36 \pm 0.3 \\
0\end{array}$ & $\begin{array}{l}10.83^{\mathrm{ab}} \pm 0.2 \\
5\end{array}$ & $\begin{array}{l}72.94^{\mathrm{ab}} \pm \\
0.56\end{array}$ & $\begin{array}{l}0.34 \pm 0.00 \\
4\end{array}$ & $\begin{array}{l}67.99 \pm 0 . \\
29\end{array}$ & $\begin{array}{l}11.33^{\mathrm{ab}} \pm \\
0.16\end{array}$ \\
\hline $\mathrm{FC}$ & $73.56 \pm 0.59$ & $0.34 \pm 0.004$ & $\begin{array}{l}66.83 \pm 0.2 \\
8\end{array}$ & $11.72^{a} \pm 0.23$ & $\begin{array}{l}74.28^{\mathrm{ab}} \pm \\
0.51\end{array}$ & $\begin{array}{l}0.35 \pm 0.00 \\
5\end{array}$ & $\begin{array}{l}67.54 \pm 0 . \\
29\end{array}$ & $\begin{array}{l}11.28^{\mathrm{ab}} \pm \\
0.18\end{array}$ \\
\hline FO & $73.61 \pm 0.36$ & $0.33 \pm 0.004$ & $\begin{array}{l}66.92 \pm 0.3 \\
2\end{array}$ & $10.44^{\mathrm{b}} \pm 0.20$ & $\begin{array}{l}72.33^{\mathrm{ab}} \pm \\
0.63\end{array}$ & $\begin{array}{l}0.35 \pm 0.00 \\
6\end{array}$ & $\begin{array}{l}67.44 \pm 0 . \\
36\end{array}$ & $\begin{array}{l}11.11^{\mathrm{ab}} \pm \\
0.14\end{array}$ \\
\hline $\mathrm{FCO}$ & $75.22 \pm 0.74$ & $0.33 \pm 0.005$ & $\begin{array}{l}66.74 \pm 0.3 \\
6\end{array}$ & $\begin{array}{l}11.28^{\mathrm{ab}} \pm 0.2 \\
5\end{array}$ & $\begin{array}{l}73.11^{\mathrm{ab}} \pm \\
0.53\end{array}$ & $\begin{array}{l}0.35 \pm 0.00 \\
4\end{array}$ & $\begin{array}{l}67.87 \pm 0 . \\
32\end{array}$ & $\begin{array}{l}11.39^{\mathrm{ab}} \pm \\
0.18\end{array}$ \\
\hline$P$ & 0.088 & 0.060 & 0.073 & 0.001 & 0.014 & 0.868 & 0.088 & 0.007 \\
\hline
\end{tabular}

a,b: Means with different superscripts in each row are significantly different

an alternative molting material during feed withdrawal method and the yield impact is similar with the mixtures presented by the researchers as an alternative.

Wu et al. (28) carried out a study with two white laying hybrids to determine the impact of two different forced-molting methods on the egg yield as well as on egg quality. It was observed that the molting method did not have an impact on egg yield and egg weight. The present trial findings showed that at the start of the study (14-28 days), group $M$ egg yield was almost similar to group $F$ while at the mid of study, the egg yield of group $M$ was higher as compared to group $F$. Kuçukyilmaz et al. (20). Yilmaz and Sahan (29) study showed that $50 \%$ egg yield with $62.05 \mathrm{~g}$ average egg weight were produced from brown layers hens which were subjected to force molting with full grain and zinc oxide application. In terms of significance, the lowest egg shape index form at the end of the study was manifested by group
$K(72.06)$ while the highest values were displayed by group $M(74.89)$ which coincided with the results reported by Kucukyilmaz et al. (20) that molting had an impact on index form. The findings by the same researchers that shell thickness and Haugh unit are affected by molting. In contrast, the results of our study asserted that eggshell thickness and Haugh unit were not affected by molting and feed supplements were not commensurate. In a study carried by Bell and Kuney (8) the Haugh unit values obtained from feed withdrawal groups (74.7-75.7) are less than the values calculated for feed withdrawal groups in the trial. Regarding egg shape index, in groups $M$; there was no difference between egg shape indexes and eggshell thickness in the present study. These results was correlated to another study which observed no differences in shape index and eggshell thickness of brown layer hens during 12 days of forced molting with feed withdrawal (1). Molino et al. (22) and Bell and Kuney (8) reported that the egg- 
shell thickness is $37 \mathrm{~mm}$ after feed withdrawal as a force molting and this value was found proximal to eggshell thicknesses measured in groups $M \quad 0.35$ $\mathrm{mm}$ ). However, other researchers observed that forced molting improves eggshell thickness but our trial results were different (6). Khoshoei and Khalali (17) reported no difference in the eggshell thicknesses in the 3rd and 4th months after peak yield with different force molting methods such as feed withdrawal method, different grain applications, cottonseed bran and molting feed applications after fasting. At the end of the 22 days of the molting period, about $0 \%$ and $15.56 \%$ mortality were observed in the groups. When a comparison is made with the mortality rates reported by Biggs et al. (10) in a program without feed withdrawal $(0-2.4 \%)$, it is observed that such range of mortality is commensurate with the results of groups $F C, F O$ and $F C O$. Moreover, it is much less than the figures for group $F$ and the alfalfa flour groups. When a comparison is made with the $2.60 \%$ mortality value observed by Petek (24) in groups without feed withdrawal, the results for groups $F C, F O$ and FCO were found low while the values of group $F$ and the alfalfa flour groups were found higher than this value. The results of the study were not similar to those of Aygun and Yetisir (5) who also reported no difference between the mortality rates of the groups without feed withdrawal. This suggests that feed consumption in the groups may have been inadequate during the molting period. The conclusion made in the study that $M$ groups feed consumptions were similar to those of group $K$. However, various studies reported that molting white layer hens subjected with the application of different fasting periods increased feed consumption (20). Molino et al. (22) carried out a study in which the activities of feed withdrawal programs and feed restricted programs were compared and reported that the group averages were similar in terms of feed consumption. When the feed consumption of the 0-98 day period in the study is assessed, the results do not coincide because there are differences between the groups. However, the feed consumption value reported from the feed withdrawal group in the study is less than the values acquired in the study. Researchers report that the feed conversion ratio of 2.27 for poultry which has been observed during feed withdrawal molting period and this value is higher than the feed conversion ratios reported for groups M (2.03-2.09). These differences are attributed to the difference found between groups in which raw nutrients were used in the feed during the yield period. Keshavarz (15) reports a feed consumption of $109.2 \mathrm{~g}$ during yield period for Babcock B300 layer hens subjected to 10 days of feed withdrawal and a feed conversion ratio were found 2.55 while Bell and Kuney (8) reported that animals subjected to feed withdrawal had a feed consumption of $102.9 \mathrm{~g}-104.2 \mathrm{~g}$ and a feed conversion ratio was found 2.59-2.60. When our study findings are compared with these values, it is observed that the feed consumptions of group $M$ are higher and the feed conversion ratios are lower. A farm-based study was conducted in which feed withdrawal method were used as a force molting and molting feed contained maize without salt which is further supplemented with $P$ and Vitamins (9). The feed conversion ratios of this farm study were compared with the feed conversion ratio of our study, and it was observed that the values of groups $F$ and $M$ are less than the values reported by the researchers.

\section{Conclusion}

It is concluded from our study, that there were significant differences were observed between the groups in terms of egg production mean except FCO group and the yields of all treatment groups were found significantly higher than the control groups. It was observed that supplementing groups $M$ with Lcarnitine and Oregano individually had a positive impact on egg yield whereas using them together had a negative impact. Moreover, all feed supplements had also shown a positive impact on the egg yields of groups $A$. As a result, it can be concluded that alfalfa flour supplemented with various feed additives could be used as an alternative during feed withdrawal method. Further, the adding of L-carnitine and Oregano together into alfalfa flour has a positive impact on performance in terms of animal welfare.

Acknowledgements: This research was supported by BAPK of Afyon Kocatepe University. Project Number: 07.VF.002. The authors thank to AKÜ BAPK.

\section{References}

1. Akşit M, Yalçın S, Karul AB. Physiological stress parameters during food withdrawal period in force moulted hen. Arch Geflügelkd 2003; 67: 212-216.

2. Alodan MA, Mashaly MM. Effect of Induced Molting in Laying Hens on Production and Immune Paramaters. Poult Sci 1999; 78: 171- 177.

3. AOAC. Official Methods of Analysis of the Association of Official Analytical Chemists. 1984; $14^{\text {th }}$ ed., Inc., Arlington, Virginia.

4. Aygun A. The investigation of response of different hybrid laying hen to withdraval molting according to egg production and quality, PhD Thesis, Selçuk Univ Fen Bil Ens, Konya 2007.

5. Aygun A, Yetisir R. Researches on the responses of different hybrid layers with respect to egg production performances to forced molting programs with and without feed withdrawal. J Anim Vet Adv 2009; 8(12): 2680-86.

6. Bar A, Razaphkovsky V, Shinder D, Vax E. Alter- 
native procedures for molt induction. Practical aspects. Poult Sci 2003; 82:543-550.

7. Bar A, Razaphkovsky V, Wax E, Malka Y. Effect of age at molting on postmolting performance. Poult Sci 2001; 80: 874-878.

8. Bell DD, Kuney DR. Effect of fasting and post-fast diets on performance in molted flocks. J Appl Poult Res 1992; 1: 200-206.

9. Bell DD. An alternative molting procedure. UC research for Non-feed-removal molting. (A supplement to "Farm Evaluation of Alternative Molting Procedures": Bell, D.D., and D.R. Kuney, J Appl Poult Res 2004; 13: 673-679). [web: http : // animalscience. ucdavis. edu/avian / pip41. Pdf ]. Access: 08/2011.

10. Biggs PE, Persia ME, Koelkebeck KW, Parsons $\mathrm{CM}$. Further evaluation of nonfeed removal methods for molting programs. Poult Sci 2004; 83: 745-752.

11. Card LE, Nesheim MC Poultry Production . 11. ed., Lea and Febiger,1972, Philadelphia.

12. Carey JB, Brake JT. Induced Molting of Commercial Layers. Poult Sci and Technology Guide, 1989, No:10, NC State University, Raleigh, NC.

13. Gongruttananun N, Saengkudrua K. Responses of laying hens to induce molting procedures using cassava meal of variable length with or without recovery period. Agric Nat Res 2016; 50: 400-407

14. Gulhan T, Oztabak K, Hasret D, Toker N, Matur $E$. The effect of vitamin $E$ on cellular immune responses in laying hens forced-moulted by different methods. Arch Geflügelk 2006; 70 (1): 28-34.

15. Hurwitz S, Wax E, Nisenbaum Y, Ben Moshe M, Plavnik I. The response of laying hens to induced molt as affected by strain and age. Poult Sci 1998; 77: 22-31.

16. Keshavarz K, Quimby FW. An investigation of different molting techniques with an emphasis on animal welfare. J Appl Poult Res 2002; 11: 54-67.

17. Khan RU, Zia-ur Rahman, Javed I, Muhammad F. Effect of vitamins, probiotics and protein on semen traits in post-molt male broiler breeders. Anim Reproduct Sci 2012; 135: 85-90.

18. Khoshoei EA, Khajali F. Alternative InducedMolting Methods for continuoes Feed withdrawal and their Infiuence on postmolt performance of laying Hens. Int J Poult Sci 2006; 5(1): 47-50.

19. Koelkebeck KW, Anderson KE. Molting layers-
Alternative methods and their effectiveness. Poult Sci 2007; 86: 1260-1264.

20. Koelkebeck KW, Anderson KE. Molting layersAlternative methods and their effectiveness. Poultry Sci $2007 ; 86$ : 1260-1264

21. Kuçukyilmaz K, Erensayin C, Orhan H. The effects of different fasting time on egg production and egg internal and external quality post molted laying hen. Akdeniz Üniv Ziraat Fak Derg 2003; 16(2): 199-210.

22. Minoura $M$, Ohguchi $H$, Ito $H$, Noda $K$, Kato $Y$. Investigation on induced molting without fasting by feeding the diets which were mainly composed of the wheat bran or the deffatted rice bran in hens. Res Bulletin of the Aichi-ken Agric Res Center, 2005; 31: 173-179 (sum.)

23. Molino AB, Garcia EA, Berto DA, Pelícia K, Silva $A P$, Vercese $F$. The effects of alternative forcedmolting methods on the performance and egg quality of commercial layers. Brazilian J Poult Sci 2009;11,2: 109-113.

24. National Research Council (NRC). Nutrient requirement of poultry. 1994; $9^{\text {th }}$ Revised Edition, National Academy Pres, Washington, DC.

25. Petek M. The effect of different molting programmes on main yields in the commericial laying hen. UÜ Vet Fak Derg 2001;20: 39-44.

26. Rafeeq MI, Rashid NI, Awan MAI, Tariq MMI, Abbas FI, Ahmed ZI, Taj I. Effect of Forced Molting on Body Characteristics and Post-Molting Egg Production Performance of Layers in Quetta, Pakistan. Brazilian J Poult Sci 2013; v.15 / n.4 / 347-352.

27. Rauch W Die elastische Verformung von Hühnereiem als MaBstab fiir die Beurteilung der Schalenstabilitat. Arch Geflugelk 1965; 29: 467-477.

28. SAS JMP ${ }^{T M}$ 5.0.1a Statistical and Graphic Guide. 2002;A Business Unit of SAS 1989-2002. Cary, NC, USA.

29. Thirunavukkarasu $P$, Moorthy $M$, Viswanathan $K$ Body Weight Changes of Single Comb White Leghorn Layers at Different Ages During Induced Moult. Int J Poult Sci 2007; 6,12: 858-859.

30. Wu, G, Gunawardana P, Bryant MM, Voitle RA, Roland DA. Effect of molting methods and dietary energy on postmolt performance, egg components, egg solid, and egg quality in Bovans White and Dekalb White hens during second cycle phases two and three. Poult Sci 2007; 86 (5): 69 876. 
31. Yılmaz B, Sahan U. The Effects of Different Forced Molting Methods on Egg Production and Egg Quality in Egg Layers. 11 $1{ }^{\text {th }}$ European Poultry Conference, 2002; 06-10 September 2002, Bremen Germany. 